\title{
Nfkbie-deficiency leads to increased susceptibility to develop B-cell lymphoproliferative disorders in aged mice
}

\author{
Veronique Della-Valle $\mathbb{C}^{1,2}$, Damien Roos-Weil ${ }^{1,2,3,4}$, Laurianne Scourzic ${ }^{1,2}$, Enguerran Mouly $y^{1,2}$, Zakia Aid ${ }^{1,2}$, \\ Walaa Darwiche ${ }^{5}$, Yann Lecluse ${ }^{6}$, Frederik Damm7, Sylvie Mémet $\mathbb{0}^{8}$, Thomas Mercher ${ }^{1,2}$, Said Aoufouchi $\mathbb{1}^{9}$, \\ Florence Nguyen-Khac ${ }^{4,10}$, Olivier A. Bernard ${ }^{1,2}$ and Hussein Ghamlouch (10)
}

\begin{abstract}
Aberrant NF-KB activation is a hallmark of most B-cell malignancies. Recurrent inactivating somatic mutations in the NFKBIE gene, which encodes $\mathrm{IKB} \varepsilon$, an inhibitor of NF-KB-inducible activity, are reported in several B-cell malignancies with highest frequencies in chronic lymphocytic leukemia and primary mediastinal B-cell lymphoma, and account for a fraction of NF-KB pathway activation. The impact of NFKBIE deficiency on B-cell development and function remains, however, largely unknown. Here, we show that Nfkbie-deficient mice exhibit an amplification of marginal zone B cells and an expansion of B1 B-cell subsets. In germinal center (GC)-dependent immune response, Nfkbie deficiency triggers expansion of GC B-cells through increasing cell proliferation in a B-cell autonomous manner. We also show that Nfkbie deficiency results in hyperproliferation of a B1 B-cell subset and leads to increased NF-kB activation in these cells upon Toll-like receptor stimulation. Nfkbie deficiency cooperates with mutant MYD88 signaling and enhances B-cell proliferation in vitro. In aged mice, Nfkbie absence drives the development of an oligoclonal indolent B-cell lymphoproliferative disorders, resembling monoclonal B-cell lymphocytosis. Collectively, these findings shed light on an essential role of $\mathrm{I} B \boldsymbol{B}$ in finely tuning B-cell development and function.
\end{abstract}

\section{Introduction}

The NF- $\mathrm{kB}$ signaling pathway plays essential roles in cell survival, differentiation, proliferation, inflammation, and immune regulation ${ }^{1}$. Aberrant NF- $\mathrm{KB}$ activation is frequently observed in several B-cell malignancies ${ }^{2-6}$. This activation can result from interactions with the tumor microenvironment ${ }^{7-9}$ or as a result of somatic mutations affecting NF- $\mathrm{KB}$ family members or upstream signaling components, such as CD79B, BIRC3, CARD11, MYD88, TNFAIP3/A20, or inhibitors of kappa B (IкBs;

Correspondence: Olivier A. Bernard (olivier.bernard@inserm.fr) or Hussein Ghamlouch (hussein.ghamlouch@hotmail.com)

${ }^{1}$ INSERM U1170; équipe labélisée Ligue Nationale Contre le Cancer; Gustave

Roussy, Villejuif, France

${ }^{2}$ Faculté de Médecine, Université Paris-Sud, Université Paris-Saclay, Orsay, France

Full list of author information is available at the end of the article

These authors contributed equally: Veronique Della-Valle, Damien Roos-Weil refs. ${ }^{3,5,6,10-13}$ ). The inactivation of IKB genes is recurrently observed in human B-cell malignancies. The NF- $k B$ inhibitor I-kappa-B-epsilon (NFKBIE) gene, which encodes $\mathrm{I} \kappa \mathrm{B} \varepsilon$, is targeted by a recurrent 4-bp truncating mutation in addition to rare point mutations in $5-10 \%$ of chronic lymphocytic leukemia (CLL) ${ }^{2,3,14-16}$ and various B-cell lymphomas (BCLs), including diffuse large B-cell lymphoma (DLBCL; 5\%), mantle cell lymphoma (5\%), splenic marginal zone lymphoma (1.8\%), and primary mediastinal B-cell lymphoma (PMBCL; 23\% of patients $^{12,17}$. NFKBIE mutations are enriched among advanced stage CLL and associated with poor-prognostic outcome, suggesting that they might be involved in disease progression $^{2,3,12,17}$. Compared to NFKBIE-wild-type (WT) patients, NFKBIE-mutated CLL cells showed

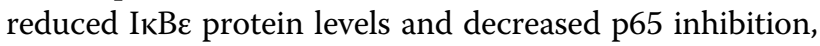


along with increased phosphorylation and nuclear translocation of p65 (ref. ${ }^{2}$ ).

The NF- $\mathrm{BB}$ transcription factor family members are maintained as inactive homo- or heterodimers in the cytoplasm by $I_{\kappa} B$, including the classical I $B$ protein

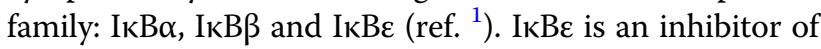
inducible NF- $\mathrm{kB}$ activity, which traps the Rel proteins in the cytoplasm at the resting state ${ }^{1,18,19}$. Its expression is higher in primary murine splenic B cells than in $\mathrm{T}$ cells and has a specific role in the late retro-control of B-cell response to external stimuli, including signaling through the $\mathrm{B}$-cell receptor $(\mathrm{BCR})$ and Toll-like receptors (TLRs) $^{20-22}$. IKB $\varepsilon$ is more expressed in IgM+B cells than in IgG $+B$ cells and presents a slower turnover than IкB $\alpha$ and $I \kappa B \beta$, following in vitro activation of primary splenic $B$ cells with LPS or a combination of anti-IgM and CD40L $\left(\right.$ ref. ${ }^{21}$ ). I $\kappa B \varepsilon$ shows preferential specificity for NF- $\kappa B$ Rel homodimers, with respect to other NF- $\mathrm{kB}$ transcription factor subunits ${ }^{21}$. I $\kappa B \varepsilon$-deficient $B$ cells present higher expression and activity of Rel at early time points of activation with IgM and IL4 compared to WT cells ${ }^{20-22}$.

To get more insights into the role of NFKBIE in normal and malignant B-cell differentiation, we studied Nfkbiedeficient mice and showed that the loss of Nfkbie results in marginal zone B (MZB) and B1 cells expansion, and a higher sensitivity to T-cell-dependent and -independent stimulation. We also show that Nfkbie deficiency cooperates with mutant MYD88 signaling and causes enhanced B-cell proliferation. In aged mice, Nfkbie absence drives development of an oligoclonal indolent Bcell lymphoproliferative disorders, resembling monoclonal B-cell lymphocytosis (MBL).

\section{Materials and methods}

Additional information can be found in the Supplemental Methods.

\section{Mice}

Inactivated Nfkbie allele on a mixed Sv129xDBA$2 \mathrm{xC} 57 \mathrm{BL} / 6 \mathrm{~J}$ background has been described previously; ${ }^{23}$ $>20$ back-crosses were performed on the C57BL/6 J background to give rise to a pure congenic $\mathrm{Nfkbie}^{-1-}$ C57BL/6 J strain. Mutant mice in this pure C57BL/6 J background were housed in the animal facility of the Gustave Roussy Institute (SCEA, Gustave Roussy, Villejuif, France), crossed with WT C57BL/6 J mice and agematched animals of selected genotypes were sacrificed at the indicated times. Bone marrow transplantation were performed as described ${ }^{24,25}$. Animal experiments were conducted in compliance with the Gustave Roussy Institutional guidelines and authorized by the Direction Départementale des Services Vétérinaires du Val de Marne.

\section{Mice immunization}

For the analysis of germinal center (GC) formation in Tcell-dependent immune response, Nfkbie-deficient and WT age-matched 2-3-month-old mice were immunized with sheep red blood cells (SRBCs; $1 \times 10^{8}$ cells per mouse) via intraperitoneal injection. Mice were sacrificed 10 days after immunization and spleens were analyzed for GC B cells and plasma cells.

For in vivo TLR9 activation, CpG ODN (Invivogen) was administered intraperitoneally at a dose of $333 \mu \mathrm{g}$ per mouse in $200 \mu \mathrm{L}$ phosphate-buffered saline. Mice were sacrificed 10 days after immunization; inguinal lymph nodes and spleens were analyzed for GC B cells.

\section{In vitro generation of induced GC B cells}

Splenic untouched resting murine $B$ cells were first isolated from 2 to 3-month-old animals using the mouse CD43 (Ly-48) B-cell isolation kit (MicroBeads, Miltenyi Biotec) according to the manufacturer's instructions (yield $>90 \% \mathrm{CD} 19+\mathrm{B} 220+$ ). MZB and FOB cells were flow-sorted as described in the "Cell isolation and culture" section. 40LB-cells expressing CD40L and producing BAFF were a kind gift of Pr. D. Kitamura ${ }^{26}$ and cultured in DMEM media (Invitrogen) with 10\% FBS and penicillin/ streptomycin.

For B-cell culture, RPMI-1640 medium (Invitrogen) was supplemented with $10 \%$ FBS, 0,055 mM 2-ME, $10 \mathrm{mM}$ HEPES, $1 \mathrm{mM}$ sodium pyruvate, 100 units $/ \mathrm{ml}$ penicillin, and $100 \mu \mathrm{g} / \mathrm{ml}$ streptomycin (invitrogen). Purified B cells $\left(110 \times 10^{3}\right.$ cells per well) were cultured in a six-well plate in the presence of $40 \mathrm{LB}$-cells $\left(0.5 \times 10^{6}\right.$ cells per dish) that had been irradiated with 80 Gy X-ray. rIL-4 $(1 \mathrm{ng} / \mathrm{ml}$; Peprotech) was added to the primary culture for 4 days. Cells were harvested at day 4 (D4) and processed for flow cytometric analysis.

\section{MBL diagnosis}

The appearance of a B220lowCD19+CD5+ population and its percentage among peripheral blood mononuclear cells (PBMCs) were used for initial MBL-like disease diagnosis. The diagnostic criterion for MBL in mice was arbitrary defined as the appearance of oligoclonal/monoclonal B220lowCD19+CD5+ cells, constituting over 5\% of PBMCs without a detectable increase in total white blood cell count.

\section{Genescan analysis}

cDNA from sorted Nfkbie-deficient B cells (B220lowCD19+) and WT B cells (B220+CD19+) was PCR amplified using $\mathrm{VH}$ family primers $(\mathrm{VH} 1, \mathrm{VH} 2, \mathrm{VH}$, VH5, VH6, and VH7 as indicated) and a common $\mathrm{JH}$ FAM-conjugated primer to assess IgH CDR3 diversity ${ }^{27}$. PCR products were run on a 3130xl sequencer (Applied 
Biosystems) and data were analyzed using the Peakscanner software (Applied Biosystems).

\section{Retroviral infection and in vivo cell transfer}

MYD88WT and that containing L265P mutation (MYD88L265P) cDNA were synthesized by GenScript and subcloned into MSCV-eGFP backbone. Viral particles and transduction procedures were described previously ${ }^{3}$.

\section{Statistical analysis}

Statistical significance of differences between the results was assessed using a two-tailed unpaired Student's $t$-test with Welch's correction, performed using Prism (GraphPad software, version 5.03). Statistically significant $p$ values: * $p$ values $<0.05$; $* p$ values $<0.01$ and $* * * p$ values $<0.005$. Error bars displayed throughout the paper represent s.e.m. or s.d. as indicated in figure legends. No statistical method was used to predetermine sample size. No blinding and no randomization of samples were applied. No data was excluded.

\section{Results}

$\mathrm{Nfkbie}^{-1-}$ mice show amplification of MZB and B1 B cells

Initial analyses of immune cells in $N f k b i e^{-1-}$ mice on a mixed genetic background showed only very subtle phenotypic consequences on thymic lymphoid T-cell subsets $^{23}$. Our analysis showed that, compared to 5-8month-old WT, aged-matched $N f k b i e^{-1-}$ mice on a pure C57BL/6 J background presented an increase in cell number and frequency of a population expressing low levels of the B220 marker (B220low) in spleen, thymus, lymph nodes, peritoneal cavity, and peripheral blood (Fig. 1a). Detailed immunophenotyping in spleen and peritoneal cavity was compatible with a B1 B-cell population: CD19+, B220low/-, CD43+, CD23-, CD21-, CD93-, IgDlow, and IgM+ (Supplementary Fig. 1a).

Analysis of CD5 expression showed that both B1a (CD5+) and B1b (CD5-) subsets were increased in spleen of $\mathrm{Nfkbie}^{-1-}$ mice, whereas only the B1a cell population augmented in peritoneal cavity (Fig. 1b). Surface IgM expression on B1 cells was higher in Nfkbie ${ }^{-l-}$ than in WT splenic cells, in contrast to peritoneal ones (Supplementary Fig. 1b). Based on their surface IgM expression levels, peritoneal cavity $\mathrm{Nfkbie}^{-1-}$ (B220lowCD19+CD5+) B1a cells were subdivided into IgMhigh and IgMlow B1a subsets (Fig. 1c). The IgMlow B1a B-cell population increased with age in $\mathrm{Nfkbie}^{-1-}$ mice (Fig. 1d).

B2 populations flow cytometry analysis revealed lower frequency and numbers of mature follicular B (FoB) cells, defined as $\mathrm{CD} 19+\mathrm{B} 220+\mathrm{CD} 23+\mathrm{CD} 21+$, and increased frequency and numbers of MZB cells, defined as CD19 $+\mathrm{B} 220+\mathrm{CD} 23$ lowCD21hi, in $\mathrm{Nfkbie}^{-/-}$mice with respect to WT (Supplementary Fig. 1d), as reported ${ }^{20}$. Nfkbie ${ }^{-l-}$ MZB cells displayed significantly higher IgM expression than WT MZB cells, unlike FoB cells WT and Nfkbie cells (Supplementary Fig. 1c).

Together, these data indicate that the absence of Nfkbie affects mature B-cell subsets differentiation and leads to expansion of MZB and B1a B cells. These B-cell subsets are known to mediate the innate functions of the $B$ lineage. Both populations are particularly sensitive to variations in NF- $\mathrm{kB}$ activity and strongly influenced by BCR specificity and strength of signaling ${ }^{28-30}$.

\section{Nfkbie deficiency affects the frequency of the B1 B-cell progenitor and the transition from transitional $B$ cells to mature $B$ cells}

We next analyzed in detail hematopoietic differentiation, including B-cell development, in the bone marrow of 2month-old KO mice. Proportions of LSK cells, myeloid (CMP, GMP, and MEP), and common lymphoid progenitor were comparable between WT, $\mathrm{Nkbie}^{+/-}$and $\mathrm{Nfkbie}{ }^{-/-}$ mice (Supplementary Fig. 2a-c). Analysis of early B-cell differentiation stages, including pre-pro-B (CD19-B220 $+\mathrm{CD} 43+)$, pre-B $(\mathrm{CD} 19+\mathrm{B} 220+\mathrm{CD} 43-)$, and pro-B $(\mathrm{CD} 19+\mathrm{B} 220+\mathrm{CD} 43+)$, showed no significant alteration of the percentages and numbers of each population (Supplementary Fig. 2d, f). Analysis of Hardy fractions did not reveal any abnormalities in $\mathrm{Nfkbie}^{-1-}$ mice either (Supplementary Fig. 2e, g), indicating that loss of Nfkbie does not impact early B-cell development in the bone marrow.

We then evaluated the frequency and numbers of B1 Bcell progenitors (Lin-CD93+CD19+B220-/low) in mutant bone marrow ${ }^{31}$. Significantly higher frequency of Lin-CD93+CD19+B220-/low cells in 2-month-old $\mathrm{Nfkbie}^{-/-}$mice were observed compared to those of WT or $\mathrm{Nfkbie}^{+/-}$mice, with a trend for $\mathrm{Nfkbie}^{-/-}$B1 progenitor cell number increases (Fig. 2a).

To check the balance between B1 and B2 cells, we analyzed their proportions in the spleen and peritoneal cavity. Percentages of total CD19+ B cells increased significantly in peritoneal cavity but remained stable in the spleen of $\mathrm{Nfkbie}^{-1-}$ mice, as compared to WT animals (Fig. 2b). Percentages of splenic and peritoneal B2 cells $(\mathrm{CD} 19+\mathrm{B} 220+)$ were also similar between both genotypes (Fig. 2b); however, percentages of B1 cells were significantly higher in $\mathrm{N} f \mathrm{kbie}^{-1-}$ mice. These data indicate that there is no shift toward the B1 cell lineage in mutant mice and that the increase in $\mathrm{B} 1$ cell numbers might result from the increase of the $\mathrm{B} 1 \mathrm{~B}$-cell progenitor cells.

We next analyzed peripheral splenic B2 B-cell populations from 2-month-old mutant mice. Immature transitional B-cells frequencies and numbers were unaffected by $N f k b i e$ deficiency, whereas decrease of mature FoB-cell population and increase of MZB cells observed in older mice were already present (Fig. 2c). Additional analyses of non-B-cell lineage did not show the reported CD44- 


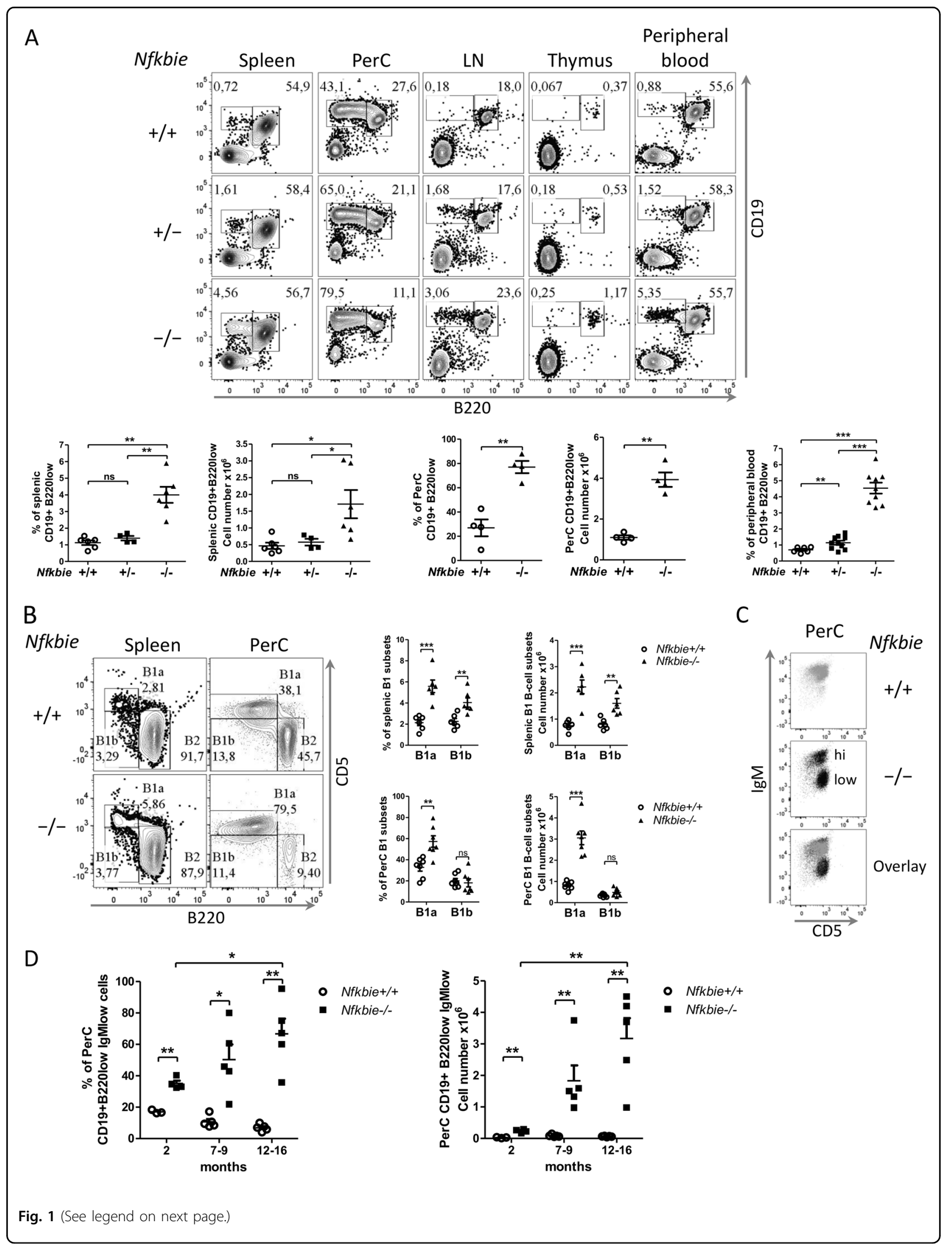


(see figure on previous page)

Fig. 1 Nfkbie ${ }^{-I-}$ mice present an expansion of B1a B cells and a biased differentiation pathway toward MZB. a Upper panel: representative plots of FACS analysis of the CD19+B220low population in spleen, peritoneal cavity (PerC), lymph node (LN), thymus, and peripheral blood of Nfkbie ${ }^{+/+}$, $\mathrm{Nfkbie}^{+/-}$, and Nfkbie ${ }^{-/-}$mice. Lower panel: percentages and absolute cell numbers of the CD19+B220low population in spleen and PerC, and percentages of the CD19+B220low population in peripheral blood. Each symbol represents one mouse. b Analysis of the B1 B-cell subset, B1a (CD19+B220lowCD5+) and B1b (CD19+B220lowCD5-), partitioning in spleen and PerC of Nfkbie ${ }^{+/+}$and Nfkbie ${ }^{-/-}$mice $(n=5)$. Each symbol represents one mouse. c Representative FACS plots of IgM and CD5 expression on the CD19+B220low cells in PerC of Nfkbie ${ }^{+/+}(n=5)$ and Nfkbie $e^{-/-}$mice $(n=5)$. $\mathbf{d}$ Percentages and absolute cell numbers of CD19+B220low IgMlow B cells in the PerC of Nfkbie ${ }^{+/+}$, and Nfkbie $e^{-/-}$of 2, 7-9 and 12-16-month-old mice $(n=5)$. Each symbol represents one mouse. Data are mean \pm SEM. ${ }^{*} p<0.05,{ }^{* *} p<0.01,{ }^{* * *} p<0.001$; ns, not significant.

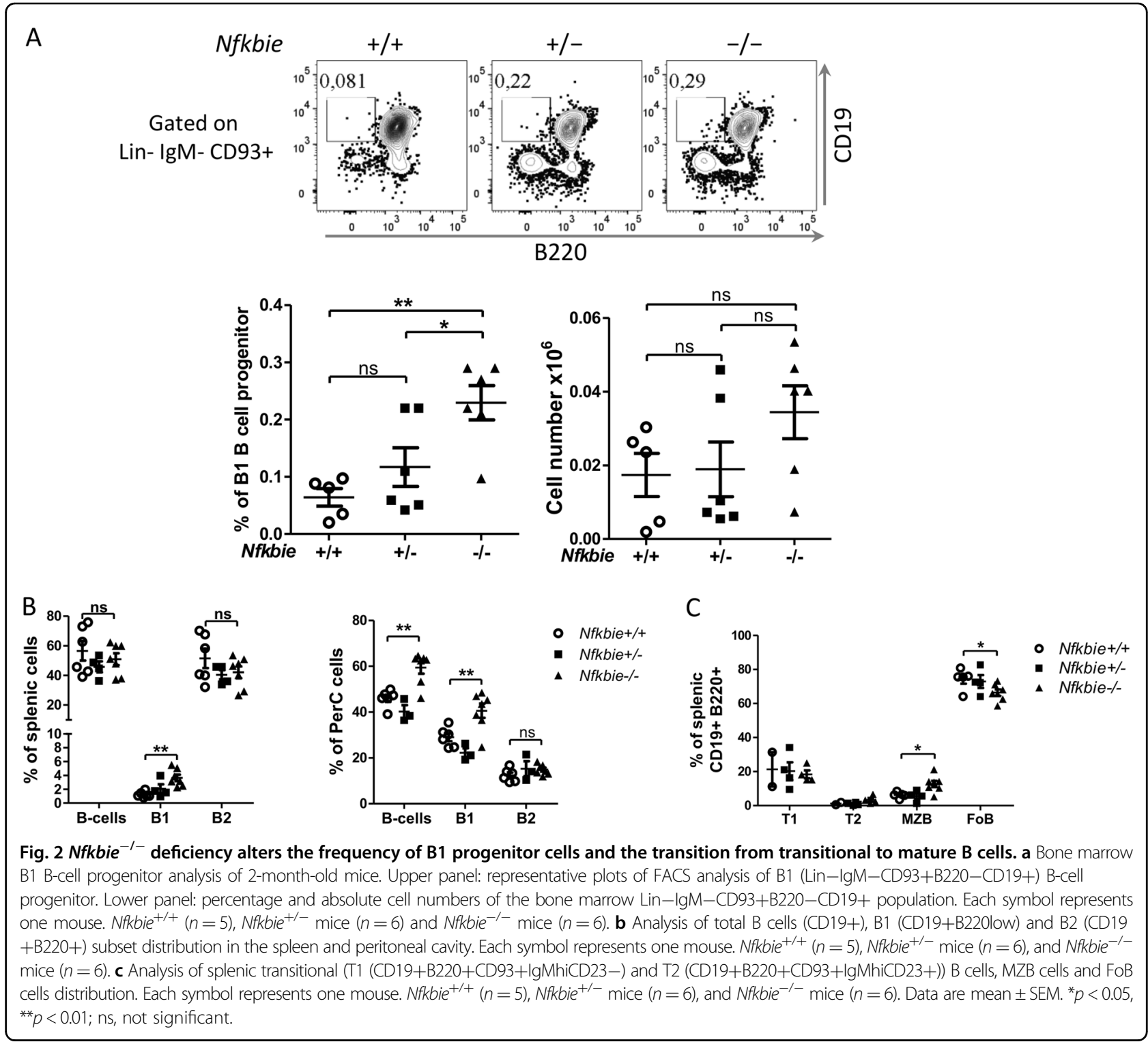

CD25+(DN3) thymocytes decrease (Supplementary Fig. 2h), which might therefore result from the mixed genetic background of the mutant mice ${ }^{23}$. No other abnormality of major hematopoietic lineages was observed in 2-month-old mice (Supplementary Fig. 2i) or older $\mathrm{Nfkbie}^{-1-}$ (Supplementary Fig. 2j) mice.

To assess whether higher B1 and MZB B-cell numbers were due to increased proliferation or reduced apoptosis, 


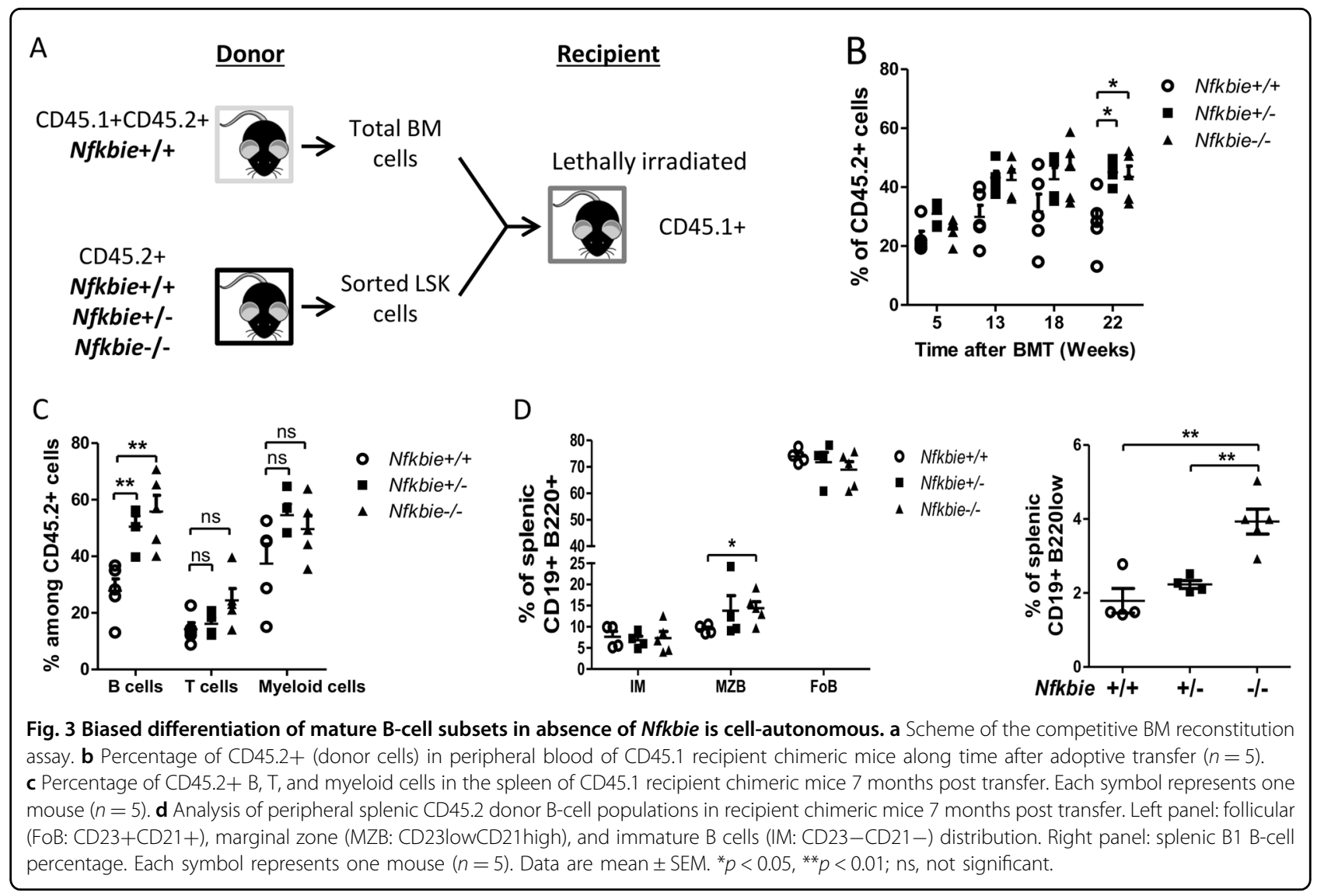

we investigated ex vivo proliferation and apoptosis. No significant differences in the proportion of Ki67+ or viable MZB, FoB, B1 B cells between Nfkbie ${ }^{-1-}$ and WT mice were detected (Supplementary Fig. 3a, b). As the frequency and cell numbers of splenic transitional B cells were equivalent between $\mathrm{Nfkbie}^{-/-}$and WT mice, these findings suggest that $N f k b i e$ deficiency biases the differentiation of transitional B cell into MZB cell fate.

Overall, these data indicate that Nfkbie is important for follicular versus MZB cell fate decision and that its loss may affect the size of the B1 B-cell progenitor compartment.

Biased differentiation toward MZB cell and expansion of B1 B-cell subsets in absence of Nfkbie is cell-autonomous

To investigate whether Nfkbie deficiency-associated changes were cell-autonomous, we performed competitive bone marrow reconstitutions (Fig. 3a for scheme). FACS analysis in peripheral blood showed that recipients of WT CD45.2+ cell had a stable reconstitution with $\sim 30 \%$ donor cells, whereas there was a steady increase in the percentage of donor cells in recipients of $\mathrm{Nfkbie}^{+/-}$ and $N f k b i e^{-l-}$ CD $45.2+$ cells that reached statistical significance at 22 weeks with over $40 \%$ of donor cells (Fig. 3b).
Analysis of the distribution of cell lineages showed that $\mathrm{Nfkbie}^{+/-}$and Nfkbie ${ }^{-1-}$ LSK (Lin-Sca1+Kit+) cell transfer resulted in a greater proportion of CD19+ B cells than that of WT cells in recipient mice (Fig. 3c). Consistent with our initial observations, at 22 weeks $N f k_{b i e^{-1-}}$ cells contributed to a significantly higher proportion of splenic MZB cells and B220lowCD19+ B1 cells than WT ones (Fig. 3d). These data establish the cell-autonomous nature of the Nfkbie-deficient-associated phenotypes.

\section{Aged Nfkbie $^{-1-}$ mice develop a MBL-like phenotype}

To assess the long-term impact of Nfkbie deficiency, we monitored monthly a cohort of ten $\mathrm{Nfkbie}^{-1-}$, ten $\mathrm{Nfkbie}^{+/-}$mice, and ten control WT mice for the presence of the B220lowCD19+ population in blood. After 12 months, five out of ten (50\%) Nfkbie $e^{-1-}$ mice started to develop MBL (defined as over 5\% of B220lowCD19+ within PBMCs; Fig. 4a). Cytological analysis of blood smears from MBL developing Nfkbie $e^{-1-}$ mice showed larger lymphocytes with more abundant cytoplasm around the nucleus (Supplementary Fig. 4a). Nfkbie -l- $^{-1}$ mice that developed MBL-like disease showed a clear expansion of CD5+ cells among the B220lowCD19+ population (Fig. 4b). Analysis of IGH gene rearrangements revealed an oligoclonal pattern of the 


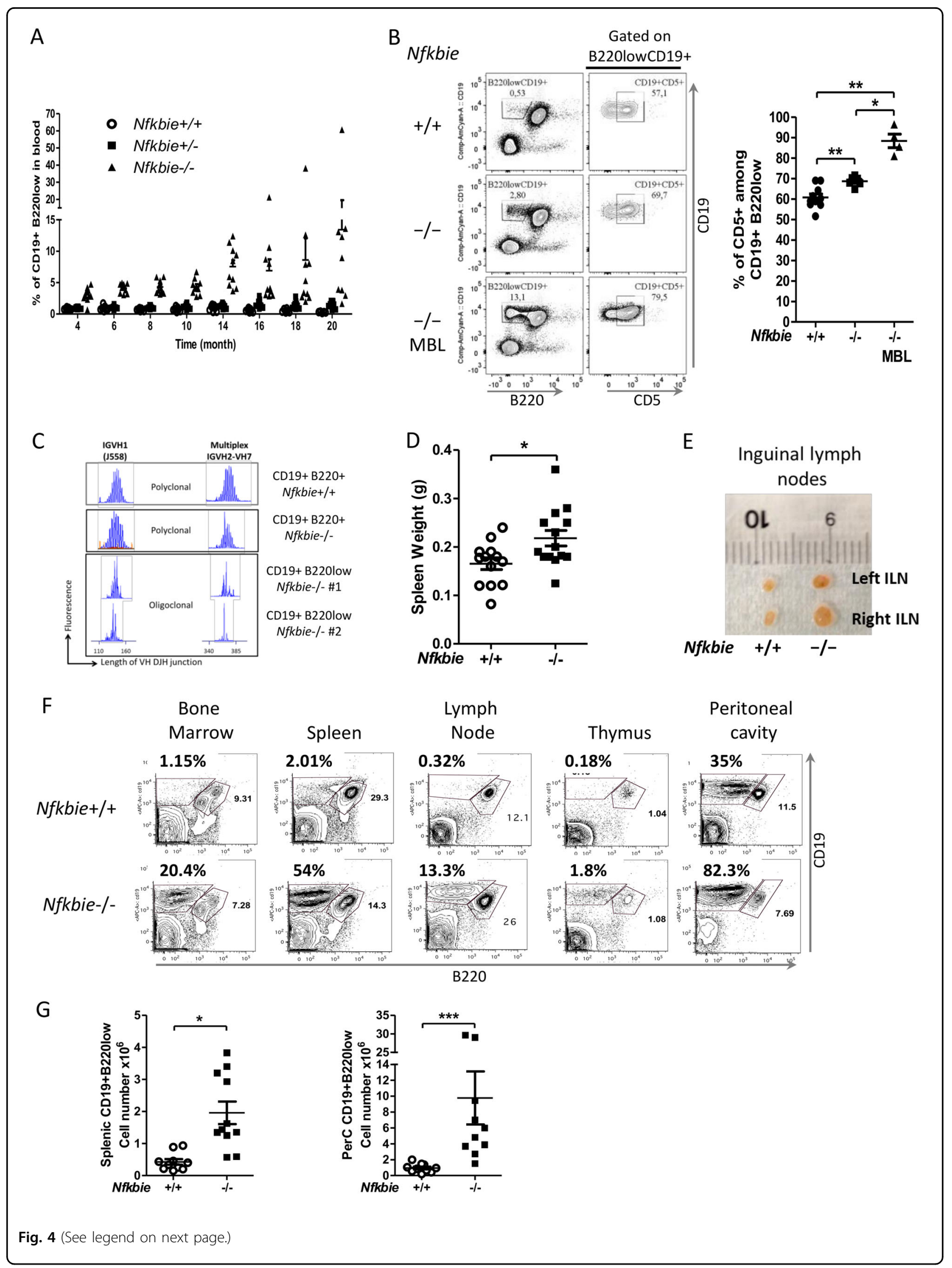


(see figure on previous page)

Fig. 4 Aged Nfkbie ${ }^{-I-}$ mice develop a MBL-like phenotype. a Peripheral blood was monitored for the presence of CD19+B220low B cells starting at 4 months. Each symbol represents one mouse $(n=10)$. $\mathbf{b}$ Right panel: percentage of CD5+ cells among CD19+B220low population in the peripheral blood of 16-month-old mice. Each symbol represents one mouse. Left panel: representative plots of FACS analysis of the CD19+B220low B cells and of the expression of CD5 on the CD19+B220low population in the peripheral blood of Nfkbie ${ }^{+/+}(n=10)$, Nfkbie ${ }^{-/-}$without MBL( $(-/-)(n=6)$, and $\mathrm{Nfkbie}^{-/-}$with MBL $(-/-\mathrm{MBL})(n=4)$. c Analyses of BCR rearrangement clonality in sorted Nfkbie ${ }^{+/+}$CD 19+B220+ B cells, and Nfkbie ${ }^{-/-}$CD19+B220+ and CD19+B220low B cells of two different 14-month-old mice (\#1 and \#2). V-J junctions were PCR amplified from cDNA of sorted B cells using VH family primers $\mathrm{VH} 1$ and multiplexed $\mathrm{PCR}$ of $\mathrm{VH} 2, \mathrm{VH} 3, \mathrm{VH} 5, \mathrm{VH} 6$, and $\mathrm{VH} 7$ ) and $\mathrm{FAM}$-conjugated consensus JH primer to assess the IgH CDR3 diversity. $\mathbf{d}$ Spleen weight of old (>12 months) Nfkbie ${ }^{+/+}(n=13)$ and Nfkbie ${ }^{-/-}$mice $(n=14)$. Each symbol represents one mouse. e Representative image from littermate $\mathrm{Nfkbie}^{+/+}$and $\mathrm{Nfkbie}^{-/-}$old mice showing enlarged inguinal lymph nodes of Nfkbie ${ }^{-1-}$ mice. $\mathbf{f}$ Representative flow cytometry profiles of CD19+B220low infiltration in bone marrow, spleen, lymph nodes, thymus, and peritoneal cavity of 18-month-old Nfkbie ${ }^{-/}$mice with MBL $(n=5)$ and age-matched $\mathrm{Nfkbie}^{+/+}$mice. $\mathbf{g}$ Absolute cell numbers of CD19+B220low B cells in the spleen and peritoneal cavity of old Nfkbie ${ }^{+/+}$and Nfkbie ${ }^{-/-}$mice $(n=11)$. Each symbol represents one mouse. Data are mean \pm SEM. ${ }^{*} p<0.05,{ }^{* *} p<0.01$.

B220lowCD19+ population in Nfkbie ${ }^{-1-}$ mice (Fig. 4c). However, total white blood cell counts were not significantly elevated in 20-month-old $\mathrm{Nfkbie}^{-1-}$ mice compared with those of control mice (Supplementary Fig. 4b) neither was survival rate of these aged mutant animals (not shown). A single $\mathrm{Nfkbie}^{-1-}$ mouse, devoid of MBL, developed a disseminated tumor in subcutaneous tissues in the cervical area, resulting in a swollen neck. Flow cytometric analysis of tumor-infiltrating cells showed a dominant population of B220+CD19+CD5B-cell population (data not shown). Nevertheless, old Nfkbie ${ }^{-1-}$ mice (>12 months) showed a mild splenomegaly (Fig. $4 \mathrm{~d}$ ) and $60 \%(6 / 10)$ of mice presented enlarged lymph nodes (Fig. 4e). They had no systemic symptoms and flow cytometry, and cell count analysis revealed significant multiorgan infiltration of B220lowCD19+ cells (Fig. 4f, g).

\section{Nfkbie-deficient B1a cells hyper-proliferate in response to TLR stimulation and exhibit enhanced NF-KB signaling}

We then investigated the impact of Nfkbie deficiency on the proliferative response of splenic and peritoneal B-cell subsets to T-cell independent stimuli, such as TLR agonists. These stimuli are known to induce NF- $\mathrm{kB}$ activity in $\mathrm{B}$ cells ${ }^{1,8,20-22,32}$. FACS-sorted splenic B-cell subsets, FoB (CD19+B220+CD23+CD21+), MZB (CD19+B220 + CD23lowCD21hi), and B1 (CD19+B220low) cells were stimulated with anti-IgM, LPS, or CpG oligodeoxynucleotides, and cell division was measured by CFSE dilution and cell count after $72 \mathrm{~h}$ of culture. We found that splenic B1 (Fig. 5a) and MZB (Supplementary Fig. 5a) cells lacking $N f k b i e$ displayed increased proliferation rate in response to LPS and CpG compared with WT B cells. Furthermore, $\mathrm{Nfkbie}^{-/-}$MZB cells showed significantly higher proliferation rate in response to IgM stimulation compared to WT MZB cells (Supplementary Fig. 5a). There was no significant difference in FoB cells proliferation in response to LPS, CpG, and anti-IgM between mutant and WT cells (Supplementary Fig. 5b). As WT
B1 cells, Nfkbie ${ }^{-1-}$ B1 cells did not respond to anti-IgM stimulation (data not shown). This confirms that MZB and $\mathrm{B} 1$ and to a lesser extent $\mathrm{FoB}$ cells require $\mathrm{I}_{\kappa} \mathrm{B} \varepsilon$ to limit their proliferative response in a B-cell-intrinsic manner.

We next focused our analysis on B-cell subsets from the peritoneal cavity. Peritoneal B2 B cells were sorted as CD19+B220+. Similarly to splenic FoB cells, increased proliferation of $\mathrm{Nfkbie}^{-1-}$ peritoneal B2 B-cells in response to stimuli did not reached statistical significance when compared with WT cells (Supplementary Fig. 5c).

In $\mathrm{Nfkbie}^{-1-}$ mice, peritoneal B1a B cells were sorted as two populations, B1a IgMhi and B1a IgMlow, while in $\mathrm{Nfkie}^{+/+}$mice B1a B cells were sorted as one single population expressing high levels of IgM (Fig. 1c). Analysis of IgM expression levels between $\mathrm{Nfkbie}^{+/+} \mathrm{B} 1 \mathrm{a}$ cells, $\mathrm{Nfkbie}^{-l-}$ B1a IgMhi showed no significant difference (Supplementary Fig. 5d). Similarly to WT cells, Nfkbie B1a IgMhi and IgMlow cells did not respond to anti-IgM stimulation (data not shown). Nfkbie ${ }^{-/-}$B1a IgMhi cells showed higher proliferation in response to LPS or CpG stimulation compared to WT B1a B cells (Fig. 5b), whereas $\mathrm{Nfkbie}^{-l-}$ B1a IgMlow cell response was similar to that of WT B1a B cells and significantly inferior to that of $\mathrm{Nfkbie}^{-l-}$ B1a IgMhi (Fig. 5b).

Spontaneous cell death assessment of sorted WT B1a cells, Nfkbie ${ }^{-/-}$B1a IgMhi and IgMlow subsets was quantified after $24 \mathrm{~h}$ ex vivo culture without any stimuli using sytox blue staining. A minor fraction of $\mathrm{Nfkbie}^{-1-}$ B1a IgMhi cells underwent cell death in such ex vivo culture (Fig. 5c). However, WT B1a cells and Nfkbie ${ }^{-1-}$ B1a IgMlow cells showed a significant higher death ratio than Nfkbie ${ }^{-1-}$ B1a IgMhi cells (Fig. 5c).

We next sought to investigate how ІкBe deficiency impacts NF- $\mathrm{kB}$ activation in $\mathrm{CD} 5+\mathrm{B} 1 \mathrm{a}$ B cells through analyzing phosphorylated p65 (RelA) levels and cRel nuclear localization by flow cytometry ${ }^{33,34}$. We found significantly higher levels of p65 phosphorylation and cRel nuclear translocation in LPS-stimulated $\mathrm{Nfkbie}^{-/-} \mathrm{B} 1 \mathrm{a} \mathrm{B}$ 


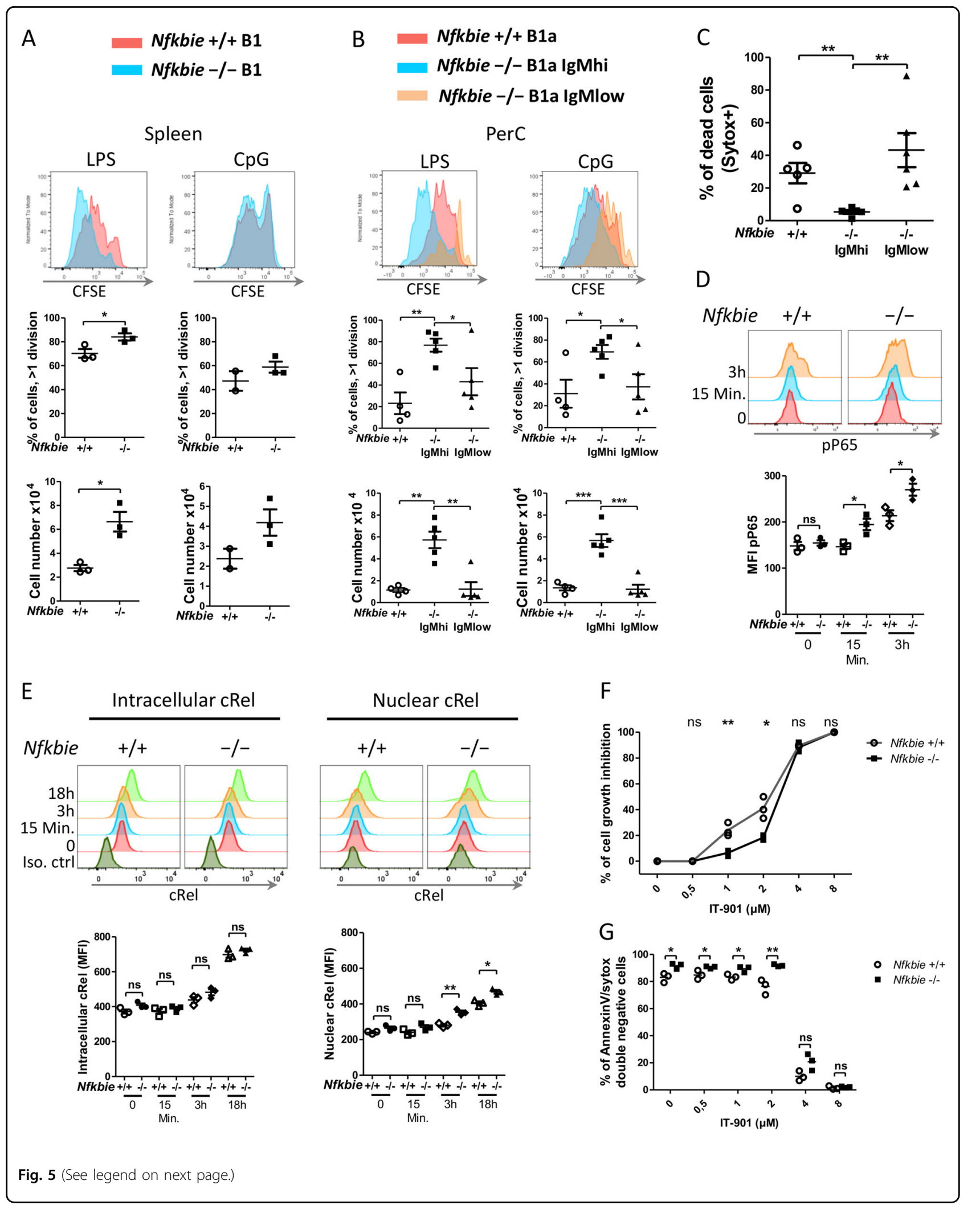


(see figure on previous page)

Fig. 5 In B cells, IKB $\varepsilon$ sets the proliferation threshold in response to TLR or T-dependent antigen stimulations. a Sorted splenic B1 cells were labeled with CFSE and cultured in the presence of LPS or CpG. Cell division was measured by CFSE dilution at $72 \mathrm{~h}$. Percentages of cells that underwent at least one division and cell numbers at $72 \mathrm{~h}$ are shown. Each symbol represents one mouse $(n=3)$. $\mathbf{b}$ Sorted peritoneal $N f k b i e^{+/+}$B1a, $\mathrm{Nfkbie}^{-1-}$ B1a lgMhi, and Nfkbie $e^{-1-}$ B1a IgMlow cells were labeled with CFSE, cultured in the presence of LPS or CpG, and analyzed as in a. Each symbol represents one mouse. Nfkbie ${ }^{+/+}(n=4), \mathrm{Nfkbie}^{+/-}$mice $(n=5)$, and Nfkbie ${ }^{-/-}$mice $(n=5)$. c Sorted peritoneal Nfkbie ${ }^{+/+} \mathrm{Bla}_{1} \mathrm{Nfkbie}{ }^{-/-}$B1a IgMhi, and Nfkbie ${ }^{-/-}$Bla IgMlow cells were cultured in complete medium without any stimulant for $24 \mathrm{~h}$. Spontaneous cell death was assessed by sytox staining. Each symbol represents one mouse. Nfkbie ${ }^{+/+}(n=4), \mathrm{Nfkbie}^{+/-}$mice $(n=5)$, and Nfkbie ${ }^{-/-}$mice $(n=5)$. $\mathbf{d}$ Analysis of phosphorylated p65 (Ser536) in sorted Nfkbie ${ }^{-/-}$and Nfkbie ${ }^{+/+}$B1a B cells at T0 and after treatment with LPS for 15 min and 3 h. Each symbol represents one mouse $(n=3)$. e Analysis of intracellular and nuclear localization of cRel in sorted B1a Nfkbie ${ }^{-/-}$and Nfkbie ${ }^{+/+}$B cells at T0 and after treatment with LPS for $15 \mathrm{~min}, 3 \mathrm{~h}$, and $18 \mathrm{~h}$. To show nuclear localization of cRel, cytoplasm was removed from cRel-stained cells with nuclear isolation media (HBSS media with $1 \mathrm{mM}$ EDTA and 0.5\% NP-40). Each symbol represents one mouse $(n=3)$. f Sorted Nfkbie ${ }^{-/-}$and Nfkbie $e^{+/+}$B1a B cells were stimulated with LPS and treated for $72 \mathrm{~h}$ with serial dilutions of IT-901. At $72 \mathrm{~h}$ viable cell number was determined by cell count and trypan blue exclusion assay. The percentage of inhibition was calculated relative to vehicle-treated cells number. 0 stands for vehicle (DMSO). Each symbol represents one mouse $(n=3)$. $\mathbf{g}$ Sorted Nfkbie $e^{-/-}$and Nfkbie ${ }^{+/+}$B1a B cells were stimulated with LPS and treated for $72 \mathrm{~h}$ with serial dilutions of IT901. Apoptosis was evaluated by Annexin V-PE and sytox staining. Percentages of double-negative (i.e., Annexin V-negative and sytox-negative) living cells are shown. 0 stands for vehicle (DMSO). Each symbol represents one mouse $(n=3)$. Data are mean \pm SEM. ${ }^{*} p<0.05,{ }^{* *} p<0.01$, ${ }^{* * *} p<0.001$.

cells compared to those of their WT counterparts (Fig. 5d, e). No difference was observed between $\mathrm{Nfkbie}^{-/-}$ and WT B1a B cells in the expression levels of cRel (Fig. 5e) and p65 (not shown) in quiescent cells or after LPS stimulation. These data indicate that $\mathrm{I} \kappa \mathrm{B} \varepsilon$ deficiency in $\mathrm{CD} 5+\mathrm{B} 1 \mathrm{a} \mathrm{B}$ cells leads to increased NF- $\mathrm{KB}$ activation upon stimulation.

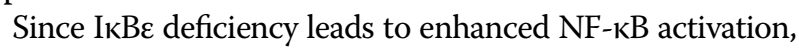
we hypothesized that Nfkbie-deficient B1a B cells would be differentially sensitive to NF- $\mathrm{kB}$ inhibition. We thus treated $\mathrm{Nfkbie}^{-1-}$ and WT B1a B cells with increasing concentration of the NF-kB inhibitor IT-901 (ref. ${ }^{34}$ ), in presence or absence of LPS stimulation. Cells were analyzed for the growth inhibition and apoptosis induction. We found that IT-901 inhibited cell growth of both $\mathrm{Nfkbie}^{-/-}$and WT B1a $B$ cells in a dose-dependent manner; however, the IC50 value was higher for $N f k b i e^{-1-}$ B1a B cells $(3 \mu \mathrm{M})$ compared to that of WT B1a B cells $(2.4 \mu \mathrm{M}$; Fig. $5 \mathrm{f})$. At $4 \mu \mathrm{M}$, no significant difference in cell growth inhibition was observed (Fig. 5f). Analysis of apoptosis showed a significant difference in survival between $N f k b i e^{-1-}$ and WT B1a B cells when cells where vehicle treated or IT-901 treated at $0.5 \mu \mathrm{M}, 1 \mu \mathrm{M}$, and $2 \mu \mathrm{M}$, whereas such difference was lost at $4 \mu \mathrm{M}$ and $8 \mu \mathrm{M}$ (Fig. $5 \mathrm{~g}$ ). These data show that $N f k b i e^{-1-}$ $\mathrm{B} 1 \mathrm{a} \mathrm{B}$ cells are generally less sensitive to NF- $\mathrm{kB}$ activity inhibition than WT B1a B cells.

Overall these data demonstrate that $\mathrm{I} \kappa \mathrm{B} \varepsilon$ is critical to limit B-cell proliferation in response to stimuli through the control of p65 phosphorylation and cRel nuclear translocation. They also establish a link between IgM expression levels and proliferation of $N f k b i e$-deficient B cells.

\section{Nfkbie deficiency enhances GC B-cell proliferation}

$\mathrm{GC}$ reaction is necessary for maturation of the humoral immune response, including production of high-affinity plasma cells and memory B-cells. We explored the impact of Nfkbie absence in GC by immunizing mice with SRBCs. FACS analysis revealed an increase in both percentages and absolute cell numbers of GC B cells in $\mathrm{Nfkbie}^{-1-}$ mice compared with those of WT mice (Fig. 6a). Frequency of follicular $\mathrm{T}$-helper cells remained similar in both genotypes (data not shown). In Nfkbie ${ }^{-1-}$ mice, IgG1+ GC B cells were significantly increased and IgM+ GC B cells were significantly decreased (Fig. 6b). Percentages but not absolute cell numbers of plasmablasts/plasma cells were reduced in $N f k b i e^{-1-}$ mice compared to those of WT mice (Fig. 6c). Nfkbie $e^{-1-}$ mice had significantly higher percentages and cell numbers of GL7-IgG1+ memory B cells (Fig. 6d). These data suggest that $\mathrm{I} \kappa \mathrm{B} \varepsilon$ plays a role in the generation/proliferation of $\mathrm{GC} \mathrm{B}$ cells, isotype switching, and terminal differentiation of GC B cells.

To establish the cell-autonomous nature of this phenotype, we sorted naive B cells from $N f k b i e^{-/-}$and $N f k b i e^{+/+}$ mice, and compared their capacity to generate GC B cells in vitro under culture conditions that mimic the GC reaction $^{26}$ (Fig. 7a for scheme). To avoid the confounding effect of Nfkbie deficiency in biasing mature B-cell subsets repartition, we sorted $B$ cells as separate FoB and MZB populations. At day 4 (D4), we observed a significant increase in the total cell numbers of $\mathrm{Nfkbie}^{-1-}$ FoB cells compared to $N f k b i e^{+/+}$FoB cells (Fig. 7b); however, differences between $N f k b i e^{-1-}$ and $N f k b i e^{+/+}$MZB cells did not reach statistical significance (Fig. 7b). Flow cytometry analysis showed significantly increased numbers of in vitro-induced GC B cells (iGCB; CD19+GL7+CD95+) derived from $\mathrm{Nfkbie}^{-/-}$vs $\mathrm{Nfkbie}^{+/+}$FoB cells but not for MZB cells at D4 (Fig. 7c). In vitro-induced plasmablast (iPB) population (CD19+CD138+) was higher in $\mathrm{Nfkbie}^{-/-}$ compared to $N f k b i e^{+/+}$MZB cells at D4 (Fig. 7c).

We further investigated iGCB cell number changes in this system by analyzing cell cycle and apoptosis. iGCB cells derived from $N f k_{b i e^{-/-}}$FoB cells displayed a 


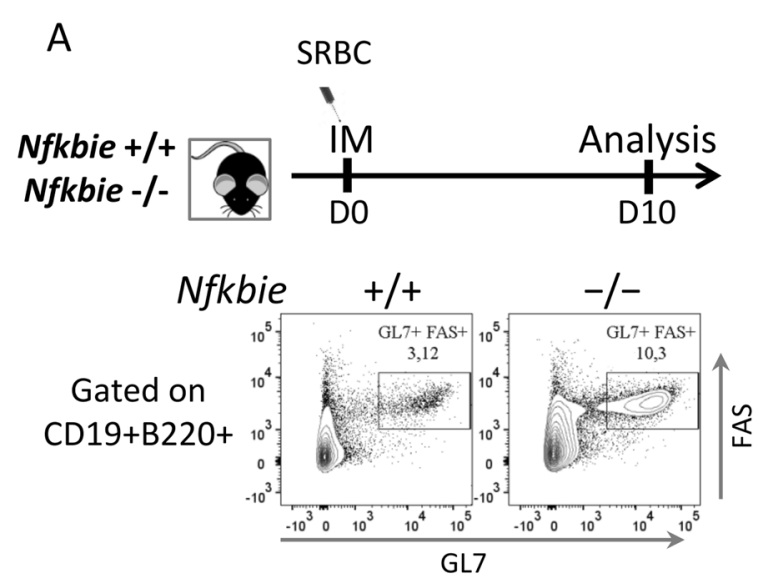

B
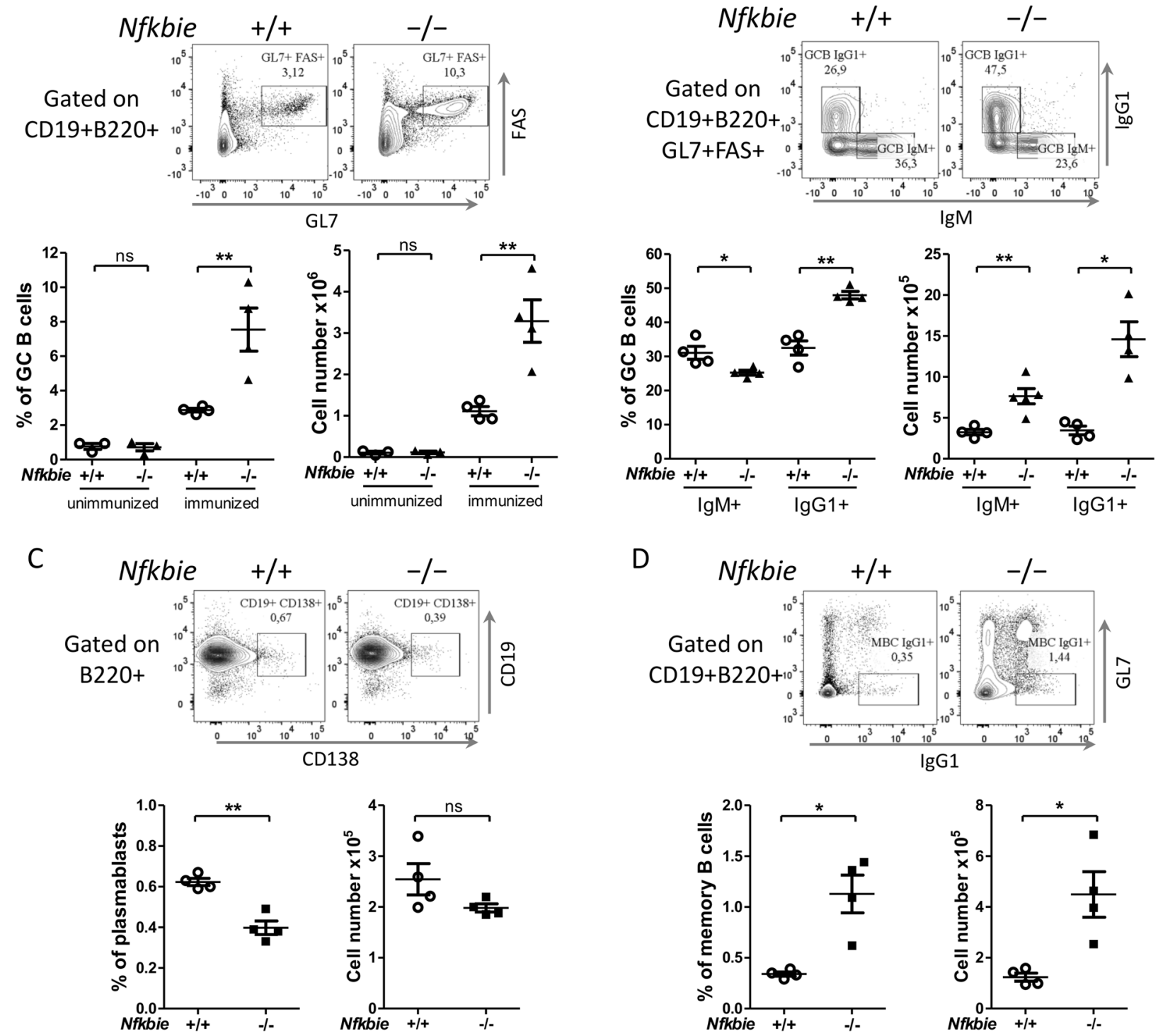

Fig. 6 Nfkbie deficiency enhances GC B-cell formation. a Scheme of the immunization protocol. Mice were intraperitoneally immunized with SRBC on day 0 (D0) and analyzed 10 days afterward (D10). Representative flow cytometry analysis of splenocytes from SRBC-immunized mice of the indicated genotypes. Gates depict germinal center (GC) B cells (B220+CD19+CD95(Fas)+GL7+). Percentages and absolute cell numbers of GC B cells in the spleen of immunized mice are shown. Each symbol represents one mouse $(n=4)$. $\mathbf{b}$ Flow cytometry plots showing IgM and IgG1 expression by GC (B220+CD19+CD95(Fas)+GL7+) B cells. Histograms show percentages of lgG1+ and lgM+Nfkbie ${ }^{+/+}$, and Nfkbie ${ }^{-\prime-}$ GC B cells. Each symbol represents one mouse $(n=4)$. c Splenic plasma cells generation after SRBC immunization of Nfkbie $e^{+/+}$and $\mathrm{Nfkbie}^{-/-}$mice; shown as FACS profiles (upper panel) and quantitation (lower panel). Each symbol represents one mouse $(n=4)$. $\mathbf{d}$ Memory $B$ cells formation after SRBC immunization of $\mathrm{Nfkbie}^{+/+}$and $\mathrm{Nfkbie}{ }^{-/-}$mice; shown as FACS profiles (upper panel) and quantitation (lower panel). Each symbol represents one mouse $(n=4)$. Data are mean \pm SEM. ${ }^{*} p<0.05,{ }^{* *} p<0.01 ;$ ns, not significant.

significant increase of $\mathrm{S}$ and $\mathrm{G} 2 / \mathrm{M}$ phases as compared to iGCB cells derived from $\mathrm{Nfkbie}^{+/+}$FoB cells (Fig. 7d). No such difference was observed for MZB cells (Fig. 7d).
Analysis of the apoptosis rate showed no difference between $\mathrm{Nfkbie}^{-1-}$ and $\mathrm{Nfkbie}^{+/+}$in both FoB and MZB cells subsets (Fig. 7e). 


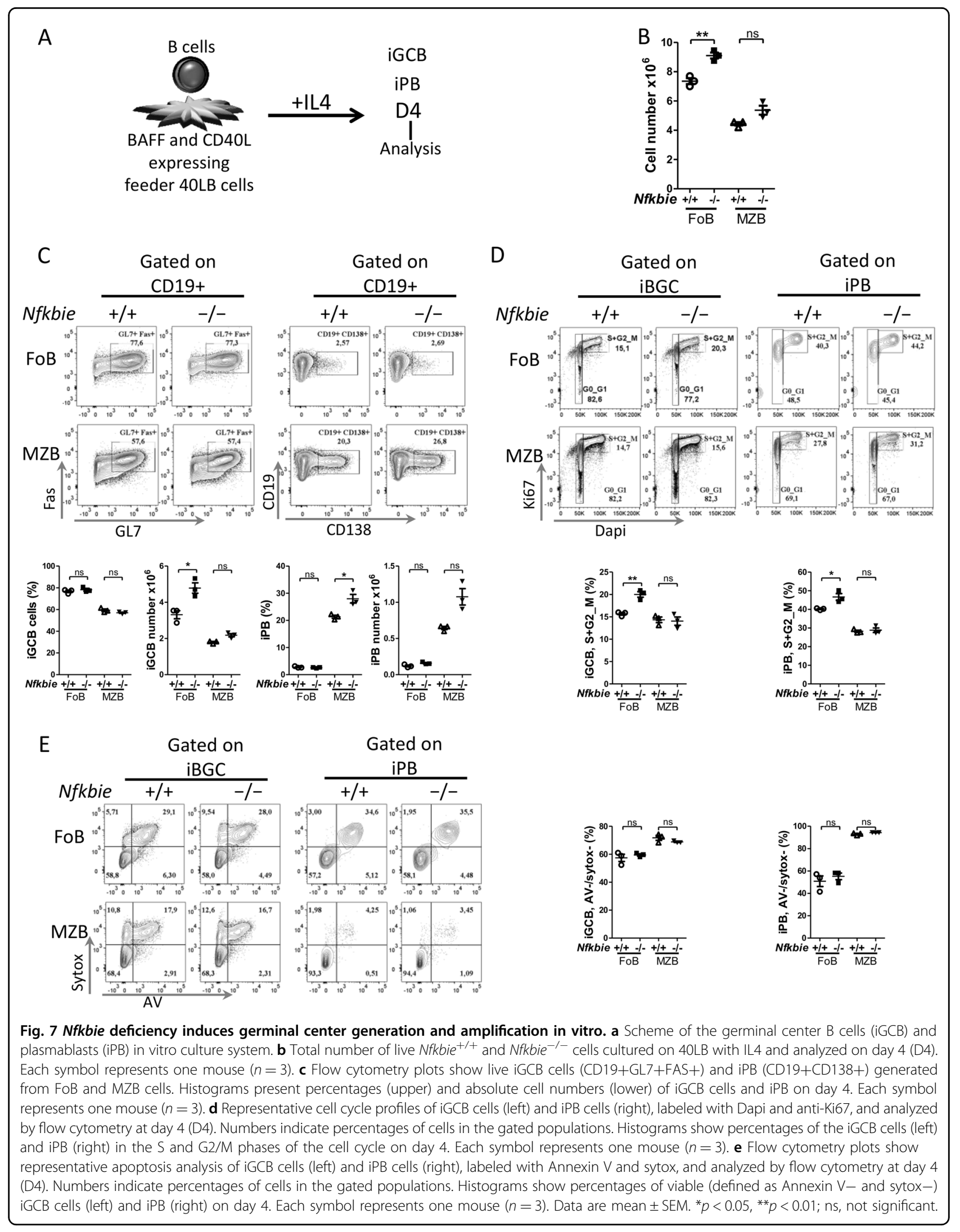


Table 1 Co-occurrence of NFKBIE and MYD88 mutations in several human B-cell malignancies.

\begin{tabular}{lllll}
\hline $\begin{array}{l}\text { Number of } \text { MYD88-mutated } \\
\text { patient }\end{array}$ & $\begin{array}{l}\text { Number of NFKBIE-mutated } \\
\text { patient }\end{array}$ & $\begin{array}{l}\text { Number of } \text { MYD88 and NFKBIE- } \\
\text { mutated patient }\end{array}$ & B-cell neoplasm Reference \\
\hline 172 & 37 & 5 & DLBCL & Reddy et al. ${ }^{11}$ \\
8 & 4 & 1 & rDLBCL & Morin et al. ${ }^{17}$ \\
1 & 1 & 1 & PCDLBCL-LT $\quad$ Fox et al. ${ }^{37}$ \\
15 & 27 & 1 & CLL & Nadeu et al. ${ }^{39}$ \\
16 & 1 & 1 & WM & Roos-Weil et al. \\
\hline
\end{tabular}

$D L B C L$ diffuse large B-cell lymphoma, $r D L B C L$ relapsed diffuse large B-cell lymphoma, $P C D L B C L-L T$ primary cutaneous diffuse large B-cell lymphoma-leg type, $C L L$ chronic lymphocytic leukemia, WM Waldenström macroglobulinemia.

These in vitro data mirror the in vivo results and indicate that Nfkbie deficiency in B cells induces expansion of GC B cells through increasing proliferation of GC B cells in a B-cell-autonomous fashion.

To demonstrate the direct impact of Nfkbie deficiency on proliferation via cell cycle, we used a CRISPR/Cas9 approach and successfully generated three knockout Nfkbie clones of the pro-B-cell line Ba/F3 (Supplementary Fig. 6a). We confirmed the knockout of Nfkbie gene at the protein level in the three clones (Supplementary Fig. 6a). Proliferation survival and cell cycle analysis were performed in control and edited cells in the presence of IL-3 at $24 \mathrm{~h}, 48 \mathrm{~h}$, and $72 \mathrm{~h}$. Nfkbie knockout clones exhibited increased proliferation when compared to WT cells (Supplementary Fig. 6b). Nfkbie knockout clones showed a decrease in the percentages of cells in G0/G1 phase, and an increase in $\mathrm{S}$ and $\mathrm{G} 2 / \mathrm{M}$ phases as compared to controls (Supplementary Fig. 6c). Analysis of apoptosis failed to detect any changes between knockout and WT cells (Supplementary Fig. 6d). Altogether, these data indicate that the effect of Nfkbie deficiency on proliferation of $\mathrm{Ba} /$ F3 cells is due to an increased cell cycle entry and is apoptosis independent.

\section{Nfkbie loss cooperates with MYD88 signaling}

MYD88 codes for an proximal signaling adaptor downstream of IL-1R and mammalian TLRs. Mutations in MYD88, essentially the missense p.L265P mutation, are observed in a wide range of B-cell malignancies ${ }^{8,10,13,35-39}$. They have been shown to activate the JAK and NF-kB pathways $^{36}$. Since both NFKBIE and MYD 88 mutations may control TLR signaling and co-occur in human B-cell malignancies (Table 1), we used mutant MYD88 to document the interactions between MYD88 and Nfkbie mutations in primary B cells. Purified WT or Nfkbie $e^{-1-}$ follicular (CD19+B220+CD23+CD21+) B cells were activated with anti-IgM and anti-CD40, transduced with MYD88 (WT) or MYD88L265P (LP) retrovirus, and cultured with anti-CD40 for $36 \mathrm{~h}$ and the proportion of fluorescent cells was monitored in the culture, as described $^{40}$ (Fig. 8a for scheme). As already reported ${ }^{40}$, expression of mutant MYD88 conferred a growth advantage over B cells expressing WT constructs. However, ectopic expression of MYD88LP conferred a significant growth advantage to the Nfkbie-deficienttransduced cells in comparison to other genotypes (Fig. 8b). Nfkbie-deficient-MYD88LP-transduced cells showed an increased cell number compared to other conditions (Fig. 8c). This indicates that targeting the TLRNFKB axe endows the double-mutant cell with a growth advantage over the other cells.

MYD88 is the canonical adaptor for signaling pathways downstream of the members of the TLR family, including TLR9. Genetic or pharmacological inactivation of TLR9 was shown to inhibits MYD88L265P-induced proliferation in murine and human B-cell lines ${ }^{40,41}$. TLR9 is an essential component of the oncogenic BCR signaling supercomplex (MyD88-TLR9-BCR) identified in ABCDLBCL and leads to NF- $\mathrm{kB}$ activation ${ }^{41}$. Therefore, to examine the cooperation between Nfkbie loss and MYD88 signaling in vivo, we immunized mice intraperitoneally with the TLR9 ligand, CpG oligodeoxynucleotides, and analyzed inguinal lymph nodes 5 days later for GC B-cell formation. We observed a significant higher GC B-cell frequency and absolute cell numbers in Nfkbie ${ }^{-/-}$ compared to $N f k b i e^{+/+}$mice (Fig. 8d). In the spleen, there was no induction of GC B-cell generation as the percentage of GC B cells was similar to unimmunized mice (data not shown). These data indicate that signaling through TLR9/MYD88 may cooperate with Nfkbie deficiency to promote GC B-cell formation and proliferation.

\section{Discussion}

The prevalent inactivation of NFKBIE in human BCL and the link between polymorphisms in the NFKBIE gene, and human autoimmune and infectious diseases ${ }^{42,43}$ suggest a role for $\mathrm{I} \kappa \mathrm{B} \varepsilon$ in B-cell development, function, and transformation. To address this question, we analyzed in detail young and aging Nfkbie-deficient mice. Our data indicate that $\mathrm{I}_{\kappa} \mathrm{B} \varepsilon$ activity is required for correct B-cell development on the one 


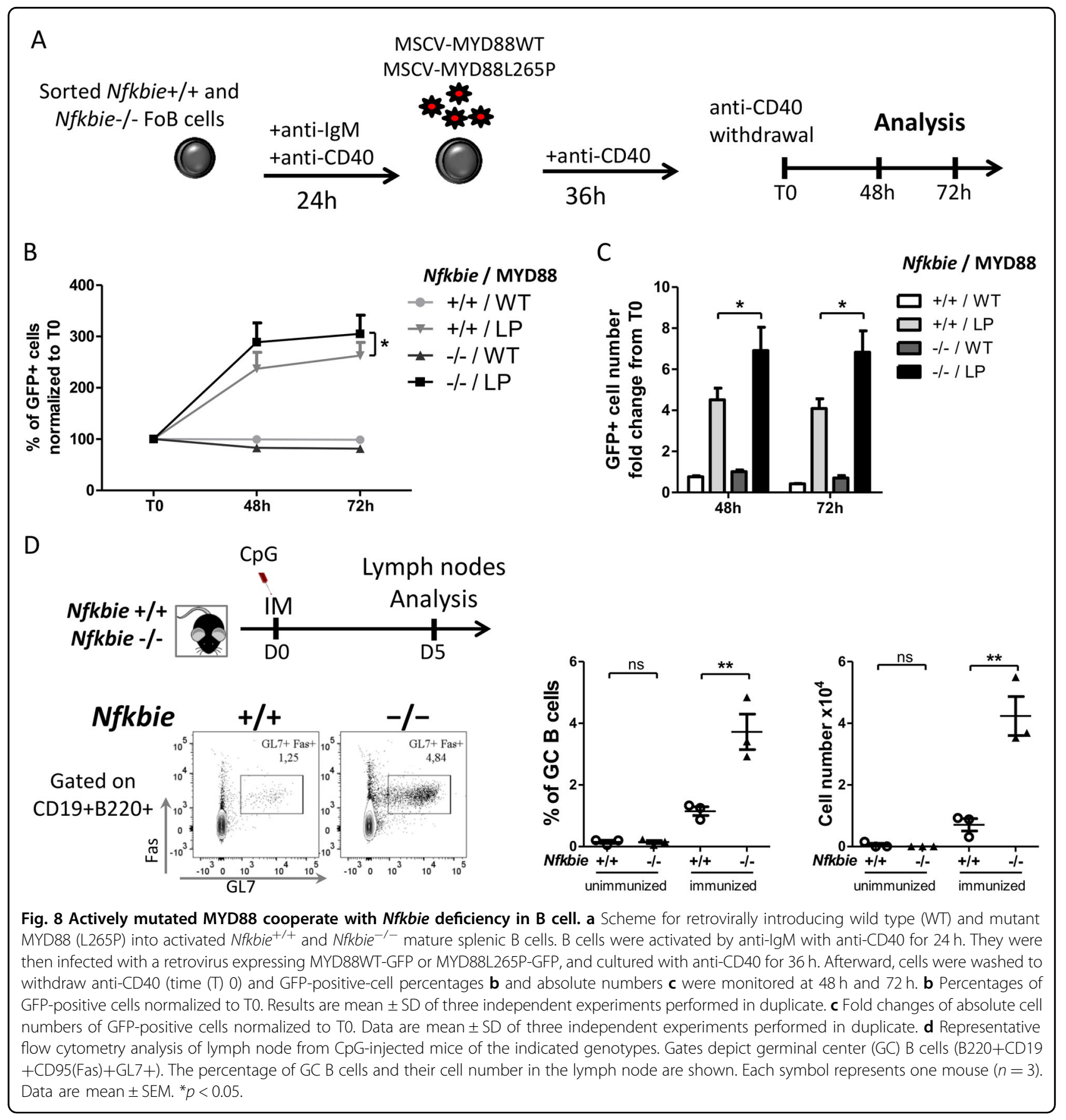

hand, and for limiting B-cell activation induced by stimuli triggered by pathogens, such as TLR ligands and for restricting proliferation of GC B cells on the other hand. In addition, Nfkbie deficiency induces MZB cell amplification in line with previous observations ${ }^{20}$-due to a higher differentiation of transitional $B$ cells toward MZB cells over the

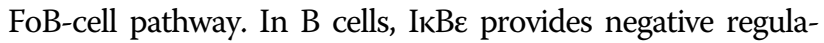
tion upon TLR stimulation by controlling NF- $\mathrm{kB}$ activation through limiting nuclear translocation of Rel-containing NF$\kappa B$ dimers (p65 and $c \operatorname{Rel})^{2,20-22}$. In LPS-stimulated I B deficient CD5+B1a B cells, we observed stronger p65 phosphorylation and cRel nuclear translocation, suggesting enhanced NF- $\mathrm{KB}$ activity in these cells. This was further supported by NF- $\mathrm{kB}$ activity inhibition experiments, which uncovered a decreased sensitivity to drug-induced apoptosis and cell proliferation inhibition of I $\mathrm{KB} \varepsilon$-deficient B1a B cells, with respect to their WT counterparts.

NF- $\mathrm{KB}$ activity downstream $\mathrm{BCR}$ and cytokine receptors signaling plays critical roles in the commitment of transitional B cells into follicular or MZB cell fate ${ }^{29}$ and in the 
development of mature B1 B cells ${ }^{28,30}$. Mice deficient in components of the alternative NF- $\mathrm{kB}$ pathway (e.g., NF$\kappa B 2$ ) or of the classical NF- $k B$ pathway (e.g., NF- $k B 1$ and Rel), present a drastic reduction in the number of FoB and MZB cells, and B1 B cells, respectively ${ }^{28}$. In keeping with its negative regulator function ${ }^{20,21}$, absence of Nfkbie elicits elevated NF- $\mathrm{kB}$ activity downstream BCR and cytokine receptors ${ }^{2,20-22}$ that likely accounts for the bias in mature B-cell development observed here.

We also uncovered that Nfkbie activity is required for correct development and function of B1 B cells. B1 cells might differentiate from fetal liver and adult bone marrow B1 progenitors ${ }^{31}$. The bone marrow of $\mathrm{Nkkbie}^{-/-}$mice contains more B1 progenitors than WT mice. However, we did not observe increased proliferation or survival of the B1 B cells directly isolated from $\mathrm{Nfkbie}^{-1-}$ and WT mice. These findings suggest a role for I $\mathrm{KB} \varepsilon$ in the generation or expansion of $\mathrm{B} 1$ progenitor pool. $\mathrm{BCR}$ signaling plays an instructive role in B1a cell lineage determination and maintenance $e^{28,30,44,45}$. In addition, recent studies showed that BCR signaling cooperates with TLR signaling in controlling expansion and activation of B1a B cells ${ }^{46-49}$. NF-kB is one of the key transcription factors activated by $B C R$ and TLR signaling. Consequently, increase of NF- $\mathrm{KB}$ activation upon BCR and TLR signaling likely drives the expansion of B1a cell compartment observed in the Nfkbie-deficient mice.

Consistent with IкBe being required to limit B-cell activation $^{20,22}$, our findings revealed that Nfkbie deficiency enhances proliferation of MZB and B1a IgMhi B cells in response to the TLR4 and TLR9 ligand, LPS, and CpG oligodeoxynucleotides respectively. B1a cells of Nfkbiedeficient mice show a significant downregulation of IgM expression in peritoneal B1a cells, with respect to WT and presented two B220lowCD19+CD5+B1a populations with different levels of IgM expression (B1a IgMhi and B1a IgMlow). Nfkbie ${ }^{-/-} \mathrm{B} 1 \mathrm{a}$ IgMlow population showed impaired proliferation response to TLRs stimuli and reduced ex vivo survival compared to B1a IgMhi cells, in agreement with the key role of BCR in B1 cells expansion and activation ${ }^{28,30,45-49}$. Downregulation of IgM on B cells contributes to a physiological B-cell immune tolerance mechanism called anergy, and serves to restrain their response to endogenous/self-antigens and to chronic BCR stimulation ${ }^{50}$. Our results strongly suggest that the B1a IgMlow population is phenotypically and functionally anergic as defined by in vivo downregulation of surface IgM expression, CD5 expression, in vitro hyporesponsiveness to TLR-dependent stimuli and ex vivo-reduced survival ${ }^{50-54}$. Importantly, these properties are hallmark features of human CLL ${ }^{44,54}$, in which NFKBIE is mutated in $~ 10 \%$ of cases $^{2,3,15,16}$. Recent work has uncovered cooperation between BCR and TLR in response to innate mitogenic stimuli. BCR-deficient $B$ cells show impaired proliferation in response to $\mathrm{LPS}$ or $\mathrm{CpG}$, indicating that correct $\mathrm{BCR}$ surface expression and signaling are critical for B-cell proliferation in response to such innate immune stimuli ${ }^{48,49}$. However, whether regaining IgM surface expression reverses anergic functions in these cells, as reported for autoreactive anergic cell model studies ${ }^{55}$, needs to be tested.

Nfkbie-deficient mice develop several features of human lymphoproliferative B-cell disorders, in particular, MBL, a low-penetrance premalignant stage ${ }^{13,56}$. Firstly, Nfkbie deficiency in mice leads to the expansion of an oligoclonal CD19 $+\mathrm{CD} 5+\mathrm{B}$-cell subset, and in humans, mature CD19+CD5 $+\mathrm{B}$ cells, show high transcriptional and functional similarity to CLL B cells and are candidate for being a normal cellular counterpart of CLL B cells ${ }^{13,45,54}$. Secondly, Nfkbie-deficient B1a cells exhibited increased survival, proliferation, and enhanced NF- $k B$ activity in response to stimulation consistently with the observations made for NFKBIE-mutated human CLL B-cells ${ }^{2}$. Thirdly, B220lowCD19+CD5+B cells expanded dramatically in the peripheral blood of old Nfkbiedeficient mice and infiltrated several organs, including spleen and lymph nodes. Finally, the lymphoproliferative disease developed by Nfkbie-deficient mice is indolent and has low penetrance, resembling the mostly indolent course of MBL and CLL, diseases of elderly population. Aging is associated with activation of innate immune cells, including a subset of B1a B cells, due to the increased auto- and pathogen-derived antigen availability, age-associated immune dysfunction, and age-induced gut permeability ${ }^{45,57,58}$. These age-associated changes could participate in the generation of MBL-like and/or CLL-like malignancies ${ }^{45,54,59}$. In this perspective, the indolent MBL development in $\mathrm{Nfkbie}^{-1-}$ mice might largely be attributed to the increased survival and hyperproliferation of B1a IgMhi B cells, in response to immune stimulation that would be more available with age and the anergic nature of B1a IgMlow B cells and their hyporesponsiveness to stimulation. Indeed, in mice, MBL and CLL develops from B1a CD5+B cells and several study demonstrate a crucial role for BCR expression, reactivity, and signaling in the malignant transformation of these cells ${ }^{45,60-62}$.

NF- $\mathrm{kB}$ activity plays an important role in the regulation of GC reaction ${ }^{32,63}$. Conditional deletion of Rel and Rela in GC B cells revealed that Rel is required to maintain GC populations, whereas Rela promoted Blimp-1-mediated plasma cell development ${ }^{63}$. Our data show that under Tcell-dependent immunization condition, Nfkbie deficiency promotes the excessive generation of GC B cells but not TFH cells in vivo. The amplification of GC B cells seems to be caused by an increased proliferation of Nfkbiedeficient GC B cells, suggesting a role for ІкB $\varepsilon$ in controlling proliferation of GC B cells. In line with these findings, it was recently shown that activating mutations in CARD11 (observed in 5\% of DLBCL (ref. ${ }^{5}$ )), a NF-kB activator protein downstream $\mathrm{BCR}$ signaling, increases GC formation and GC B-cell proliferation through 
enhanced cycling ${ }^{64}$. GC B cells represent the origin of several BCLs including DLBCL (ref. ${ }^{32}$ ). Deregulated NF$\mathrm{KB}$ signaling is a hallmark of GC-derived B-cell malignancies ${ }^{3,5,6,8}$. Mutations in NFKBIE gene are detected in $\sim 5 \%$ of DLBCL and $23 \%$ of PMBCL (refs. ${ }^{12,17}$ ). Our results provide a functional link between $N f k b i e$ mutations and GC-derived BCL. As pointed above, the absence of Nfkbie induces a bias of B2 B-cell development toward MZB cells. MZB B cells are the normal cellular counterpart of splenic marginal zone lymphoma, a BCL in which NFKBIE is also mutated in $\sim 3 \%$ of cases $^{12}$. Our data strongly suggest that $\mathrm{I} \kappa \mathrm{B} \varepsilon$ plays an essential role in regulating proliferation and differentiation of the B cells from which these lymphomas derive.

In CLL and several BCL, aberrant NF- $\mathrm{kB}$ activation is the result of genetic alterations in pathways leading to $\mathrm{NF}-\mathrm{kB}$ activation and aberrant stimulation from the microenvironment (through BCR, CD40, and TLR $)^{2,3,5,6,8,10-12,32}$. In vivo injection of the TLR9 ligand, CpG oligodeoxynucleotide, increased generation, and proliferation of $\mathrm{Nfkbie}^{-1-} \mathrm{GC}$ B cells compared to that of WT ones. Accordingly, MYD88L265P mutation, a mutation observed across many subtypes of human lymphoid malignancies, increased proliferation of $\mathrm{Nfkbie}^{-/-}$cells compared to that of WT B cells in vitro. In line with these findings, in Waldenström macroglobulinemia and DLBCL human cell lines, inactivation of TNFAIP3 (encoding A20, a negative regulator of NF- $\mathrm{kB}$ signaling) also cooperates with MYD88LP mutation to enhance NF- $\mathrm{kB}$ activation and resistance to $\mathrm{BCR}$ signaling inhibitor, ibrutinib ${ }^{65,66}$. Together, these observations demonstrate that inactivation of Nfkbie in mice predisposes to MBL and CLL, and upon collaboration with oncogenic events, it might promote BCL development.

\section{Acknowledgements}

We thank the members of the Gustave Roussy Platforms, including Patrick Gonin for excellent mouse care, and Philippe Rameau for assistance with FACS cell sorting. We thank Dr. Daisuke Kitamura for $40 \mathrm{LB}$ cell line. This work is supported by the INSERM, Institut National du Cancer (2013-PLBIO-09, 2016PLBIO-068 and INCa-DGOS-INSERM 12551), Fondation pour la Recherche Médicale (FRM), Association Laurette Fugain and équipe labelisée Ligue Nationale Contre le Cancer. S. Aoufouchi is supported by Comité Val-d'Oise de la Ligue contre le cancer. W. Darwiche is supported by a Fondation de France postdoctoral fellowship (No 00078457). H. Ghamlouch is a recipient of grants from Region lle de France, Fondation ARC pour la recherche sur le cancer and Fondation de France (No 00067113).

\footnotetext{
Author details

'INSERM U1170; équipe labélisée Ligue Nationale Contre le Cancer; Gustave Roussy, Villejuif, France. ${ }^{2}$ Faculté de Médecine, Université Paris-Sud, Université Paris-Saclay, Orsay, France. ${ }^{3}$ Sorbonne Université, Service d'hématologie clinique, Hôpital Pitié-Salpêtrière, APHP, Paris, France. ${ }^{4}$ INSERM U1138, Centre de Recherche des Cordeliers, Sorbonne Université, Paris, France. ${ }^{5}$ EA4666 Lymphocyte Normal - Pathologique et Cancers, HEMATIM, Université de Picardie Jules Verne, and Laboratoire d'Hématologie, Centre Hospitalier Universitaire Amiens-Picardie, Amiens, France. ${ }^{6} \mathrm{PFIC}$, Integrated Biology Core Facility, UMS 23/3655, Université Paris-Saclay, Gustave Roussy, Villejuif, France. ${ }^{7}$ Charité-Universitätsmedizin Berlin, corporate member of Freie Universität Berlin, Humboldt-Universität zu Berlin, and Department of Hematology,
}

Oncology, and Tumor Immunology, Berlin Institute of Health, Berlin, Germany. ${ }^{8}$ Aix Marseille Univ, CNRS, INSERM, CIML, Centre d'Immunologie de MarseilleLuminy, Marseille, France. ${ }^{9}$ CNRS UMR8200; équipe labélisée Ligue Nationale Contre le Cancer; Gustave Roussy, Université Paris-Saclay, Villejuif, France.

${ }^{10}$ Service d'Hématologie Biologique, Sorbonne Université, Hôpital PitiéSalpêtrière, APHP, Paris, France

\section{Author contributions}

VDV, DRW, FNK, OAB and HG designed the overall study and interpreted data. VDV, DRW, LS, EM, YL, WD, ZA, FD, SM, TM, SA and HG performed, analyzed and interpreted the experiments. VDV and HG generated figures. DRW, VDV, OAB and $\mathrm{HG}$ wrote the manuscript with input from SM.

\section{Conflict of interest}

The authors declare that they have no conflict of interest.

\section{Publisher's note}

Springer Nature remains neutral with regard to jurisdictional claims in published maps and institutional affiliations.

Supplementary Information accompanies this paper at (https://doi.org/ 10.1038/s41408-020-0305-6).

Received: 22 November 2019 Revised: 17 February 2020 Accepted: 26 February 2020

Published online: 13 March 2020

\section{References}

1. Zhang, Q., Lenardo, M. J. \& Baltimore, D. 30 Years of NF-kappaB: a blossoming of relevance to human pathobiology. Cell 168, 37-57 (2017).

2. Mansouri, L. et al. Functional loss of IkappaBepsilon leads to NF-kappaB deregulation in aggressive chronic lymphocytic leukemia. J. Exp. Med. 212, 833-843 (2015).

3. Damm, F. et al. Acquired initiating mutations in early hematopoietic cells of CLL patients. Cancer Discov. 4, 1088-1101 (2014).

4. Davis, R. E., Brown, K. D., Siebenlist, U. \& Staudt, L. M. Constitutive nuclear factor kappaB activity is required for survival of activated B cell-like diffuse large B cell lymphoma cells. J. Exp. Med. 194, 1861-1874 (2001).

5. Compagno, M. et al. Mutations of multiple genes cause deregulation of NFkappaB in diffuse large B-cell lymphoma. Nature 459, 717-721 (2009).

6. Okosun, J. et al. Integrated genomic analysis identifies recurrent mutations and evolution patterns driving the initiation and progression of follicular lymphoma. Nat. Genet. 46, 176-181 (2014).

7. Herishanu, Y. et al. The lymph node microenvironment promotes B-cell receptor signaling, NF-kappaB activation, and tumor proliferation in chronic lymphocytic leukemia. Blood 117, 563-574 (2011).

8. Mansouri, L., Papakonstantinou, N., Ntoufa, S., Stamatopoulos, K. \& Rosenquist, R. NF-kappaB activation in chronic lymphocytic leukemia: a point of convergence of external triggers and intrinsic lesions. Sem. Cancer Biol. 39, 40-48 (2016).

9. Oppezzo, P. \& Dighiero, G. "Role of the B-cell receptor and the microenvironment in chronic lymphocytic leukemia". Blood Cancer J. 3, el 49 (2013).

10. Schmitz, R. et al. Genetics and pathogenesis of diffuse large B-cell lymphoma. N. Engl. J. Med. 378, 1396-1407 (2018).

11. Reddy, A. et al. Genetic and functional drivers of diffuse large B cell lymphoma. Cell 171, 481-494 e415 (2017).

12. Mansouri, L. et al. Frequent NFKBIE deletions are associated with poor outcome in primary mediastinal B-cell lymphoma. Blood 128, 2666-2670 (2016).

13. Ghamlouch, H., Nguyen-Khac, F. \& Bernard, O. A. Chronic lymphocytic leukaemia genomics and the precision medicine era. Br. J. Haematol. 178, 852-870 (2017).

14. Domenech, E. et al. New mutations in chronic lymphocytic leukemia identified by target enrichment and deep sequencing. PLOS ONE 7, e38158 (2012).

15. Rose-Zerilli, M. J. et al. Longitudinal copy number, whole exome and targeted deep sequencing of 'good risk' IGHV-mutated CLL patients with progressive disease. Leukemia 30, 1301-1310 (2016). 
16. Ljungstrom, V. et al. Whole-exome sequencing in relapsing chronic lymphocytic leukemia: clinical impact of recurrent RPS15 mutations. Blood 127, 1007-1016 (2016).

17. Morin, R. D. et al. Genetic landscapes of relapsed and refractory diffuse large Bcell lymphomas. Clin. Cancer Res. 22, 2290-2300 (2016).

18. Whiteside, S. T., Epinat, J. C., Rice, N. R. \& Israel, A. I kappa B epsilon, a novel member of the I kappa B family, controls RelA and CRel NF-kappa B activity. EMBO J. 16, 1413-1426 (1997).

19. Simeonidis, S., Liang, S., Chen, G. \& Thanos, D. Cloning and functional characterization of mouse IkappaBepsilon. Proc. Natl Acad. Sci. USA 94 14372-14377 (1997).

20. Alves, B. N. et al. IkappaBepsilon is a key regulator of B cell expansion by providing negative feedback on cRel and RelA in a stimulus-specific manner. J. Immunol. 192, 3121-3132 (2014).

21. Doerre, S. et al. Reductions in I kappa B epsilon and changes in NF-kappa B activity during B lymphocyte differentiation. J. Immunol. 174, 983-991 (2005).

22. Clark, J. M. et al. Inhibitor of kappa B epsilon (IkappaBepsilon) is a nonredundant regulator of $\mathrm{c}-$ Rel-dependent gene expression in murine $\mathrm{T}$ and $\mathrm{B}$ cells. PLOS ONE 6, e24504 (2011).

23. Memet, S. et al. IkappaBepsilon-deficient mice: reduction of one T cell precursor subspecies and enhanced lg isotype switching and cytokine synthesis. J. Immunol. 163, 5994-6005 (1999).

24. Quivoron, C. et al. TET2 inactivation results in pleiotropic hematopoietic abnormalities in mouse and is a recurrent event during human lymphomagenesis. Cancer Cell 20, 25-38 (2011).

25. Mouly, E. et al. B-cell tumor development in Tet2-deficient mice. Blood Adv. 2, 703-714 (2018).

26. Nojima, T. et al. In-vitro derived germinal centre B cells differentially generate memory B or plasma cells in vivo. Nat. Commun. 2, 465 (2011).

27. Walter, J. E. et al. Expansion of immunoglobulin-secreting cells and defects in B cell tolerance in Rag-dependent immunodeficiency. J. Exp. Med. 207, 1541-1554 (2010).

28. Pedersen, G. K., Adori, M. \& Karlsson Hedestam, G. B. NF-kappaB signaling in B1 cell development. Ann. NY Acad. Sci. 1362, 39-47 (2015).

29. Pillai, S. \& Cariappa, A. The follicular versus marginal zone B lymphocyte cell fate decision. Nat. Rev. 9, 767-777 (2009).

30. Graf, R. et al. BCR-dependent lineage plasticity in mature B cells.Science $\mathbf{3 6 3}$, 748-753 (2019)

31. Montecino-Rodriguez, E., Leathers, H. \& Dorshkind, K. Identification of a B-1 B cell-specified progenitor. Nat. Immunol. 7, 293-301 (2006).

32. Klein, U. \& Heise, N. Unexpected functions of nuclear factor-kappaB during germinal center B-cell development: implications for lymphomagenesis. Curr. Opin. Hematol. 22, 379-387 (2015).

33. Doan, L. L., Tanner, M. K. \& Grimes, H. L. Intranuclear staining of proteins in heterogeneous cell populations and verification of nuclear localization by flow cytometric analysis. J. Immunol. Methods 279, 193-198 (2003).

34. Shono, $Y$. et al. Characterization of a c-Rel inhibitor that mediates anticancer properties in hematologic malignancies by blocking NF-kappaB-controlled oxidative stress responses. Cancer Res. 76, 377-389 (2016).

35. Roos-Weil, D. et al. A recurrent activating missense mutation in waldenstrom macroglobulinemia affects the DNA binding of the ETS transcription factor SPI1 and enhances proliferation. Cancer Discov. 9, 796-811 (2019).

36. Ngo, V. N. et al. Oncogenically active MYD88 mutations in human lymphoma Nature 470, 115-119 (2011).

37. Fox, L. C. et al. Molecular mechanisms of disease progression in primary cutaneous diffuse large B-cell lymphoma, leg type during ibrutinib therapy. Int. J. Mol. Sci. 19 (2018)

38. Martinez-Trillos, A. et al. Mutations in TLR/MYD88 pathway identify a subset of young chronic lymphocytic leukemia patients with favorable outcome. Blood 123, 3790-3796 (2014).

39. Nadeu, F. et al. Clinical impact of the subclonal architecture and mutational complexity in chronic lymphocytic leukemia. Leukemia 32, 645-653 (2018).

40. Wang, J. Q., Jeelall, Y. S., Beutler, B., Horikawa, K. \& Goodnow, C. C. Consequences of the recurrent MYD88(L265P) somatic mutation for B cell tolerance. J. Exp. Med. 211, 413-426 (2014).
41. Phelan, J. D. et al. A multiprotein supercomplex controlling oncogenic signalling in lymphoma. Nature 560, 387-391 (2018).

42. Myouzen, $K$. et al. Functional variants in NFKBIE and RTKN2 involved in activation of the NF-kappaB pathway are associated with rheumatoid arthritis in Japanese. PLoS Genet. 8, e1002949 (2012).

43. Lundbo, L. F. et al. Genetic variation in NFKBIE is associated with increased risk of pneumococcal meningitis in children. EBioMedicine 3, 93-99 (2015).

44. Ntoufa, S., Vilia, M. G., Stamatopoulos, K., Ghia, P. \& Muzio, M. Toll-like receptors signaling: a complex network for NF-kappaB activation in B-cell lymphoid malignancies. Sem. Cancer Biol. 39, 15-25 (2016).

45. Hayakawa, K. et al. B cells generated by B-1 development can progress to chronic lymphocytic leukemia. Ann. NY Acad. Sci. 1362, 250-255 (2015).

46. Kreuk, L. S. et al. B cell receptor and Toll-like receptor signaling coordinate to control distinct B-1 responses to both self and the microbiota. elife 8 (2019).

47. Savage, H. P. et al. TLR induces reorganization of the lgM-BCR complex regulating murine B-1 cell responses to infections. elife 8 (2019).

48. Otipoby, K. L. et al. The B-cell antigen receptor integrates adaptive and innate immune signals. Proc. Natl Acad. Sci. USA 112, 12145-12150 (2015).

49. Schweighoffer, E., Nys, J., Vanes, L., Smithers, N. \& Tybulewicz, V. L. J. TLR4 signals in B lymphocytes are transduced via the B cell antigen receptor and SYK. J. Exp. Med. 214, 1269-1280 (2017).

50. Zikherman, J., Parameswaran, R. \& Weiss, A. Endogenous antigen tunes the responsiveness of naive B cells but not T cells. Nature 489, 160-164 (2012).

51. Goodnow, C. C., Brink, R. \& Adams, E. Breakdown of self-tolerance in anergic B lymphocytes. Nature 352, 532-536 (1991).

52. Hippen, K. L., Tze, L. E. \& Behrens, T. W. CD5 maintains tolerance in anergic B cells. J. Exp. Med. 191, 883-890 (2000).

53. Cambier, J. C., Gauld, S. B., Merrell, K. T. \& Vilen, B. J. B-cell anergy: from transgenic models to naturally occurring anergic B cells? Nat. Rev. 7, 633-643 (2007).

54. Darwiche, W., Gubler, B., Marolleau, J. P. \& Ghamlouch, H. Chronic lymphocytic leukemia B-cell normal cellular counterpart: clues from a functional perspective. Front. Immunol. 9, 683 (2018).

55. Gauld, S. B., Benschop, R. J., Merrell, K. T. \& Cambier, J. C. Maintenance of B cell anergy requires constant antigen receptor occupancy and signaling. Nat. Immunol. 6, 1160-1167 (2005).

56. Kostopoulos, I. V. et al. Clonal evolution is a prognostic factor for the clinical progression of monoclonal B-cell lymphocytosis. Blood Cancer J. 7, e597 (2017).

57. Holodick, N. E. \& Rothstein, T. L. B cells in the aging immune system: time to consider B-1 cells. Ann. NY Acad. Sci. 1362, 176-187 (2015).

58. Bodogai, M. et al. Commensal bacteria contribute to insulin resistance in aging by activating innate B1a cells. Sci. Transl. Med. 10 (2018).

59. Kikushige, Y. \& Miyamoto, T. Hematopoietic stem cell aging and chronic lymphocytic leukemia pathogenesis. Int. J. Hematol. 100, 335-340 (2014).

60. Hayakawa, K. et al. Early generated B1 B cells with restricted BCRs become chronic lymphocytic leukemia with continued c-Myc and low Bmf expression. J. Exp. Med. 213, 3007-3024 (2016).

61. Hayakawa, K. et al. Loss of a chromosomal region with synteny to human 13 q14 occurs in mouse chronic lymphocytic leukemia that originates from early-generated B-1 B cells. Leukemia 30, 1510-1519 (2016).

62. Hayakawa, K. et al. Early generated B-1-derived B cells have the capacity to progress to become mantle cell lymphoma-like neoplasia in aged mice. J. Immunol. 201, 804-813 (2018).

63. Heise, $N$ et al. Germinal center B cell maintenance and differentiation are controlled by distinct NF-kappaB transcription factor subunits. J. Exp. Med. 211, 2103-2118 (2014)

64. Wray-Dutra, M. N. et al. Activated CARD11 accelerates germinal center kinetics, promoting mTORC1 and terminal differentiation. J. Exp. Med. 215, 2445-2461 (2018).

65. Wenzl, $\mathrm{K}$. et al. Loss of TNFAIP3 enhances MYD88L265P-driven signaling in non-Hodgkin lymphoma. Blood Cancer J. 8, 97 (2018).

66. Braggio, E. et al. Identification of copy number abnormalities and inactivating mutations in two negative regulators of nuclear factor-kappaB signaling pathways in Waldenstrom's macroglobulinemia. Cancer Res. 69, 3579-3588 (2009). 